\title{
OPEN Unexpected benzene oxidation in collisions with superoxide anions
}

\author{
Carlos Guerra ${ }^{1}$, Sarvesh Kumar ${ }^{2}$, Fernando Aguilar-Galindo ${ }^{3}$, Sergio Díaz-Tendero ${ }^{4,5,6 \bowtie, ~}$ \\ Ana I. Lozano², Mónica Mendes ${ }^{2}$, Paulo Limão-Vieira² \& Gustavo García ${ }^{1,7 凶}$
}

Superoxide anions colliding with benzene molecules at impact energies from 200 to $900 \mathrm{eV}$ are reported for the first time to form massive complexes. With the aid of quantum chemistry calculations, we propose a mechanism in which a sudden double ionization of benzene and the subsequent electrostatic attraction between the dication and the anion form a stable covalently bonded $\mathrm{C}_{6} \mathrm{H}_{6} \mathrm{O}_{2}{ }^{+}$ molecule, that evolves towards the formation of benzene-diol conformers. These findings lend support to a model presenting a new high energy anion-driven chemistry as an alternative way to form complex molecules.

Benzene is one of the simplest and more stable aromatic ring molecules. It has been considered as a prototype for the study of chemical reactions involving biomolecules (pyridine ${ }^{1}$, carbamates ${ }^{2}$ ), a building block for the synthesis of carbon nano-cages ${ }^{3}$ and a precursor of hydrocarbon structures (phenol, toluene, aniline, anthracene) with important technological applications ${ }^{4}$. Oxygen superoxide anion $\left(\mathrm{O}_{2}^{-}\right)$is one of the reactive oxygen species (ROS) which is responsible for several biochemical processes leading to oxidative damage in living organisms ${ }^{5}$ and materials ${ }^{6}$. In particular, 8-oxoguanine is frequently formed by the interaction of ROS with the guanine base in DNA under conditions of oxidative stress yielding an efficient way of damaging $\mathrm{DNA}^{7}$.

Oxidative processes in benzene have been extensively studied from different points of view of scientific and technical relevance, including e.g. atmospheric reactions $s^{8,9}$, surface catalyzed reactions ${ }^{10}$ or direct mechanisms induced by metallic oxide cations ${ }^{11}$. Most of these procedures fall into the domain of room temperature chemical reactions, i.e. no significant kinetic energy of the reactants is required to trigger such processes. Yet, if the kinetic energy involved in these collisions increases, new channels are open yielding excitation, ionization or even molecular dissociation, compatible with the transferred energy. Ascenzi et al. ${ }^{12}$ showed that oxygen-benzene reactions in atmospheric pressure plasmas (corona and dielectric barrier discharges) may induce energetically disfavored chemical processes leading to formation of phenol cations $\left(\mathrm{C}_{6} \mathrm{H}_{5} \mathrm{OH}^{+}\right)$and neutral phenol $\left(\mathrm{C}_{6} \mathrm{H}_{5} \mathrm{OH}\right)$. Additionally, relevant ion chemistry processes in the interstellar medium (ISM) have been reported from lowenergy anion induced reactions with different types of hydrocarbons yielding increasingly complex molecules ${ }^{13}$.

Here we show for the first time that gas-phase energetic interactions of a superoxide anion with a benzene molecule lead to an unexpectedly and quite efficient oxidation process of the neutral molecule. Under the current experimental collision energy range probed (200-900 eV), significant neutral and cationic fragmentation of benzene is reported, whilst a comprehensive description of the underlying collision dynamics mechanisms is investigated. In addition, the experimental conditions provide binary collisions between the incoming projectile $\left(\mathrm{O}_{2}^{-}\right)$and the neutral target molecule $\left(\mathrm{C}_{6} \mathrm{H}_{6}\right)$, where no positive ions with higher mass-to-charge ratio $(\mathrm{m} / z)$ than that of the parent ion $(m / z=78 \mathrm{u})$ are expected to be formed. However, as we will discuss below, the mass/ charge analysis of the collision products reveals the presence of a prominent feature at $m / z \sim 110 \mathrm{u}$ for specific impact energies between 200 and $900 \mathrm{eV}$.

The remainder of this paper is organized as follows. In the next section we briefly present our experimental and theoretical methods, then the corresponding results are presented and discussed and finally some conclusions from this study are drawn in the last section.

\footnotetext{
${ }^{1}$ Instituto de Física Fundamental, Consejo Superior de Investigaciones Científicas, Serrano 113-bis, 28006 Madrid, Spain. ${ }^{2}$ Atomic and Molecular Collisions Laboratory, CEFITEC, Department of Physics, Universidade NOVA de Lisboa, 2829-516 Caparica, Portugal. ${ }^{3}$ Donostia International Physics Center (DIPC), Paseo Manuel de Lardizabal 4, 20018 Donostia-San Sebastián, Spain. ${ }^{4}$ Departamento de Química, Universidad Autónoma de Madrid, Módulo 13, 28049 Madrid, Spain. ${ }^{5}$ Condensed Matter Physics Center (IFIMAC), Universidad Autónoma de Madrid,

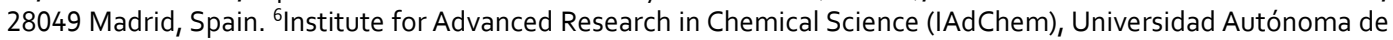
Madrid, 28049 Madrid, Spain. ${ }^{7}$ Centre for Medical Radiation Physics, University of Wollongong, Wollongong, NSW, Australia. ${ }^{\square}$ email: sergio.diaztendero@uam.es; g.garcia@csic.es
} 


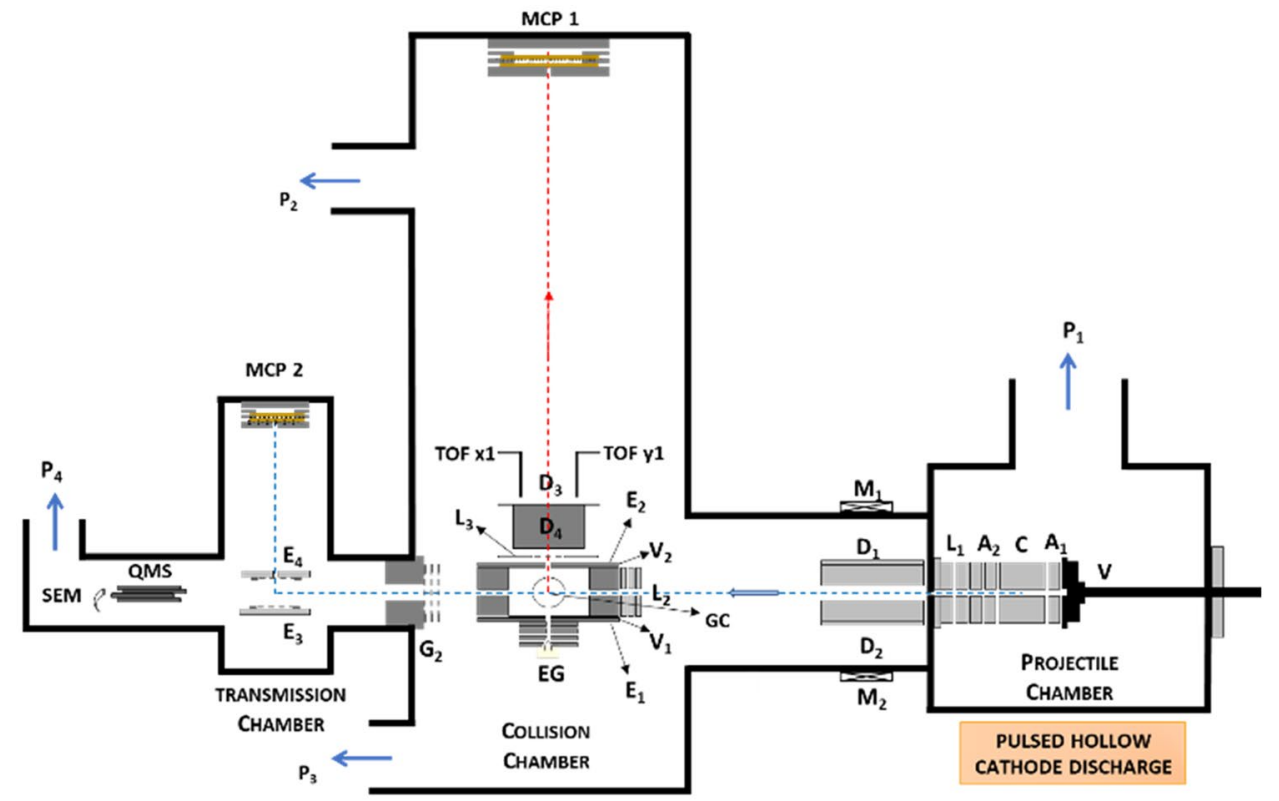

Figure 1. Schematics of the experimental setup. V, pulsed supersonic valve; C, hollow cathode discharge; A1 and A2, anodes (grounded); L1, L2, and L3, Einzel lenses; D1, D2, D3, and D4, deflecting plates; M1 and M2, magnets; E1, E2, E3, and E4, extraction plates; V1 and V2 voltage chamber; G2, retarding field analyzer grids; EG, electron gun; GC, gas cell; MCP 1 and MCP 2, multichannel plate detectors; QMS, quadrupole mass spectrometer; SEM, secondary electron multiplier detector; P1, P2, P3, and P4, turbomolecular pumps.

\section{Methods}

The experimental arrangement has been reported elsewhere ${ }^{14}$. Briefly, the primary $\mathrm{O}_{2}^{-}$beam is generated in the afterglow of a pulsed hollow cathode discharge. A schematic diagram of the experimental set-up is shown in Fig. 1.

The precursor gas projectile $\left(\mathrm{O}_{2}\right)$ is admitted into vacuum through a commercial Parker pulsed valve (VAC1250) operated at 350- $\mu$ s pulse-width in an $80-\mathrm{ms}$ duty cycle and at a gas pressure of $4.0 \times 10^{-5} \mathrm{mbar}$. It produces a supersonic expansion neutral beam that triggers the discharge when it passes along the $20 \mathrm{~mm}$ long hollow cathode which is negatively biased $(-500 \mathrm{~V})$. Positive ions initially formed in the discharge are neutralized during the plasma extinction and negative ions are finally formed by electron attachment processes. Negatively charged particles are extracted from the cathode and focused into the entrance aperture of the collision chamber (electrons are removed from the primary beam with the help of two permanent magnets placed along the beam path). The collision chamber (CC) is a $30 \mathrm{~mm}$ length metallic cube that contains the target $\mathrm{C}_{6} \mathrm{H}_{6}$ gas at a wellknown pressure (as measured with a MKS Baratron 627DX). A parallel plate system along the axis, inside the collision chamber, allows the extraction of the positive ions formed during the $\mathrm{O}_{2}-\mathrm{C}_{6} \mathrm{H}_{6}$ interactions by applying a 50-900 V pulse which is synchronized with the control unit of the supersonic valve. Positive ions enter a $1.5 \mathrm{~m}$ drift tube and their time-of-flight (TOF) is analyzed with a digital oscilloscope (Tektronix MSO 3034, 2.5 GS/s). Ion signals are formed with a microchannel plate detector (MCP-1) in single pulse mode operation with typical counting rates below $10^{2} \mathrm{~s}^{-1}$. Primary particles emerging from the CC, after the interactions, are deflected into a second $0.25 \mathrm{~m}$ length TOF spectrometer and detected by another microchannel plate detector (MCP-2). The maximum counting rate of the primary beam was typically $10^{3} \mathrm{~s}^{-1}$. The $\mathrm{C}_{6} \mathrm{H}_{6}$ pressure was maintained within 0.1-1.5 mTorr. These conditions ensure a binary collision regime and multiple scattering processes are negligible. Beneath the gas cell, an electron gun provides an energy-controlled electron beam $(0-1000 \mathrm{eV})$ entering the CC normal to the anion beam and opposite to the cation TOF mass analyzer. This electron beam is used to analyze the molecular composition of the background and the gas target as well as to provide a reference fragmentation pattern of the molecular target induced by electron impact ${ }^{15}$. The uncertainty on the point along the $2 \mathrm{~cm}$ cathode where anions are produced is spreading the arrival time distribution. This time uncertainty introduces an inherent mass resolution limitation which has been estimated to be $\Delta m / m=0.05$. Typical time of flight (TOF) mass spectra, of the primary anion beam (see Ref. ${ }^{14}$ for details), are shown in Fig. 2 for $700 \mathrm{eV}$. This figure reveals that the anion beam is mainly composed by two species assigned to $\mathrm{O}_{2}{ }^{-}$and $\mathrm{O}_{3}{ }^{-}$. Once the extraction pulse is applied, the area of the primary beam removed from the beam (see Fig. 2a) corresponds to the colliding charged species which generated the extracted ion fragments. By proper tuning the extraction pulse delay, this area can be selected all along the primary beam profile, thus allowing the choice of the projectile to collide with the target. The time scale is energy calibrated for different anion incident energies and the energy distribution corresponding to two different target conditions ( 0 and 1.3 mTorr of benzene in the scattering chamber, respectively) is plotted in Fig. 2 b. 
(a)

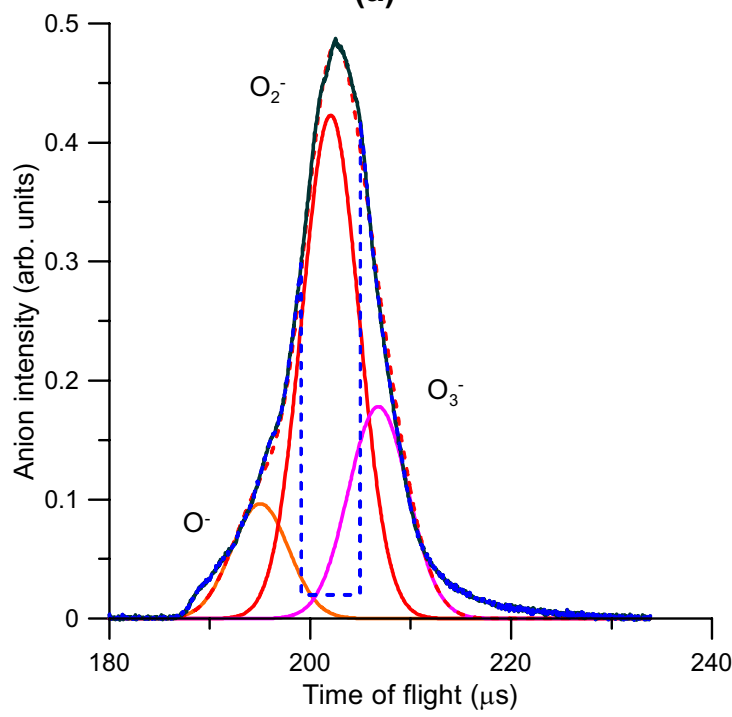

(b)

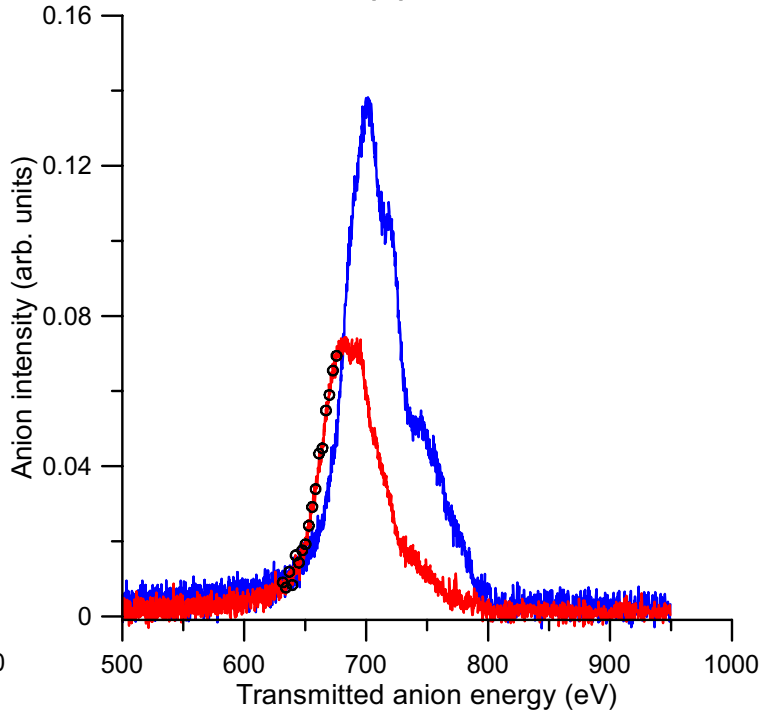

Figure 2. (a) Time of flight mass spectra of the primary anion beam: $(-) \mathrm{O}^{-},(-) \mathrm{O}_{2}^{-},(-) \mathrm{O}_{3}^{-},(--)$fraction of the beam removed by the extraction pulse (see text for details). (b) Energy spectrum of the transmitted anion intensity for (-) no target molecules in the gas cell, (-) 1.3 mTorr of benzene in the scattering chamber, (o) representative points for which the energy loss of the transmitted $\mathrm{O}_{2}{ }^{-}$anions is higher than $24.4 \mathrm{eV}$ (above the double ionization energy limit).

Calculations of the potential energy surfaces were performed in the frame of the density functional theory, in particular using the hybrid long-range corrected CAM-B3LYP functional ${ }^{16}$ in combination with the cc-pVDZ ${ }^{17}$ basis set. This functional shows a variable amount of $\mathrm{HF}$ and exchange interactions at short- and long-range and has been shown to predict exited states with better accuracy than other widely used functionals, in particular performing well for charge transfer excitations thanks to the addition of the long-range correction ${ }^{16,18,19}$. We have checked the quality of the basis set by comparing absorption spectra computed with a bigger basis, aug-cc-pVTZ, obtaining energy differences for the states of interest of less than $0.1 \mathrm{eV}$. In the exploration of the potential energy surface the critical points, i.e. minima and transition states, have been accurately located. Harmonic frequencies were computed to confirm the nature of the minima and the transition states (TSs). Furthermore, intrinsic reaction coordinate calculations were carried out to verify connectivity between TSs and adjacent minima. Harmonic frequencies were also used to correct relative energies with the Zero Point Energy (ZPE). Those simulations involving electronic excited states were performed within the time dependent DFT (TD-DFT) ${ }^{20,21}$, by using the same functional and basis set. We have also carried out a Natural Transition Orbital (NTO) analysis ${ }^{22}$ in the relevant charge transfer excited state. Details on molecular dynamics simulations are given in the Supplementary Information. All simulations have been carried out with the Gaussian 16 program ${ }^{23}$.

\section{Results and discussion}

A typical TOF spectrum of the cations extracted after the interaction of $\mathrm{O}_{2}{ }^{-}$is shown in Fig. $3 \mathrm{a}$ for $700 \mathrm{eV}$ incident energy and 1.2 mTorr of benzene in the scattering chamber ${ }^{14}$. The electron impact mass spectrum, generated in such conditions but with a $700 \mathrm{eV}$ electron beam normal to the target sample, is also shown in this figure for comparison. Both spectra are normalized to the parent ion $\left(\mathrm{C}_{6} \mathrm{H}_{6}{ }^{+}, 78 \mathrm{u}\right)$ intensity. A close inspection of this figure shows that the cation fragmentation induced by the anion beam is qualitatively the same as with the electron beam for $\mathrm{m} / \mathrm{z}$ smaller than $78 \mathrm{u}$. Notwithstanding the impact energy being the same in both cases, the collision time, projectile velocity and the energy transferred are quite different in both beams, thus lending support to the changes in the yields from both spectra. However, the main differences appear for generated molecular cation species larger than $78 \mathrm{u}$, where in Fig. $3 \mathrm{~b}$ we depict the difference between the anion and electron induced fragmentation spectra normalized to the parent ion intensity. This subtraction procedure ensures that features shown in Fig. $3 \mathrm{~b}$ are only induced by the anion beam without background contributions. In this figure, the most intense feature is found between 110 and $120 \mathrm{u}$ together with other minor contributions at 94 and $99 \mathrm{u}$.

In order to assign the 110-120 u structure's composition, we have first proved that for low pressure $(<1.5$ mTorr) conditions, only single binary collisions prevail. In this pressure range, no dependence of the measured attenuation cross-section (total electron detachment cross-section) with the gas pressure has been observed so ensuring that multiple scattering processes are negligible. In addition, we have verified that the intensity of the 110-120 u feature remains proportional to that of the parent ion for benzene pressures ranging from 0.1 to 1.5 mTorr. Under these conditions, the only possible assignment for the ion formed after collision of $\mathrm{O}_{2}{ }^{-}$and $\mathrm{C}_{6} \mathrm{H}_{6}$ with $110 \mathrm{u}$, is to consider that the projectile forms with the target a positively charged complex: $\mathrm{C}_{6} \mathrm{H}_{6} \mathrm{O}_{2}^{+}$. Yet, the quite broad nature of the 110-120 u feature shows that it can accommodate an extra contribution (126 u in 
(a)

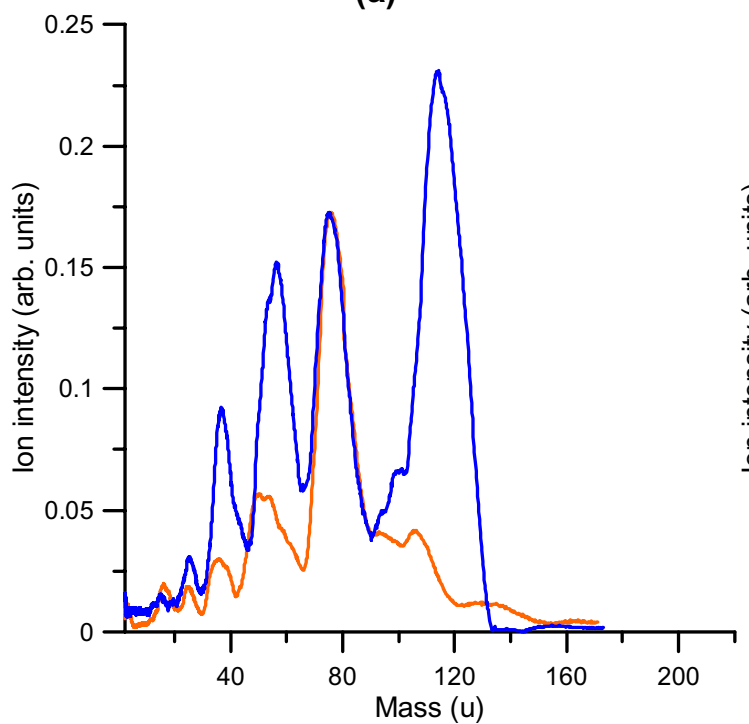

(b)

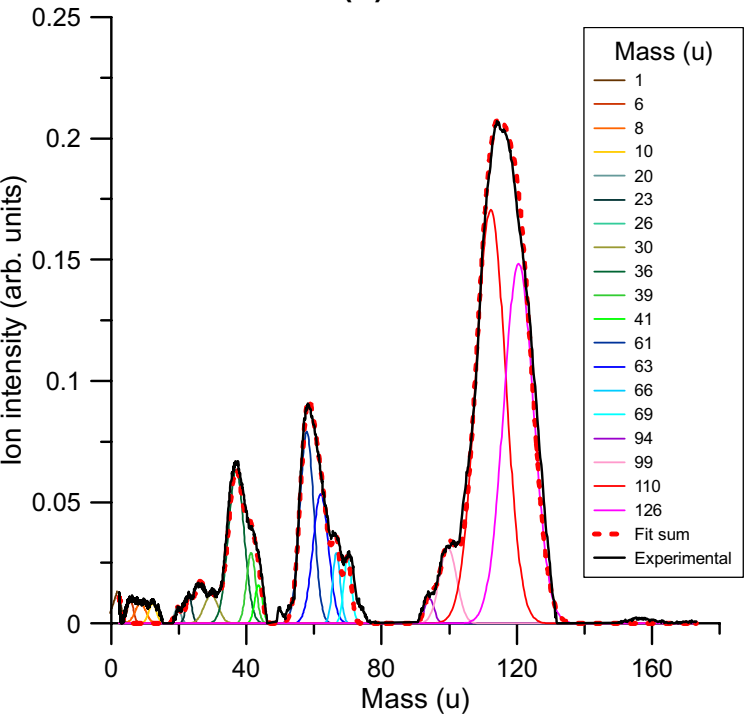

Figure 3. Positive ion spectra. (a) cationic fragmentation induced by the electron beam (-) and the negative ion (-) impact. (b) mass analysis (Gaussian function fitting) of the positive ion pattern obtained by subtracting the electron impact spectrum from that of the negative ion impact.

Fig. 3b) arising from $\mathrm{C}_{6} \mathrm{H}_{6} \mathrm{O}_{3}^{+}$formation. These weakly bound complex formations are discussed and supported with the aid of quantum chemical calculations in the next paragraphs.

$\mathrm{C}_{6} \mathrm{H}_{6} \mathrm{O}_{2}^{+}$has been previously identified as an adduct generated in air plasmas and has been proposed as a precursor for phenol production ${ }^{12}$. More prominent was the $110 \mathrm{u}$ feature observed by Tubaro et al. ${ }^{24}$ in their atmospheric pressure plasma conditions, together with $94 \mathrm{u}$ formation, the latter which was identified as being generated by the loss of an oxygen atom from the former compound according to the following mechanism:

$$
\mathrm{C}_{6} \mathrm{H}_{6}^{+\cdot}+\mathrm{O}_{2} \rightarrow \mathrm{C}_{6} \mathrm{H}_{6} \mathrm{O}_{2}^{+\cdot} \rightarrow \mathrm{O}+\mathrm{C}_{6} \mathrm{H}_{5} \mathrm{OH}^{+\cdot}
$$

Although the experimental circumstances of atmospheric plasmas and single collision events in high vacuum are categorically different, it is important to analyze possible reaction mechanisms leading to these species within the current experimental conditions and with the help of theoretical calculations on the underlying mechanisms dictated by the collision dynamics.

For this purpose, we have performed quantum chemical calculations exploring different potential energy surfaces along the reaction coordinate system, i.e. $\left[\mathrm{C}_{6} \mathrm{H}_{6} \ldots \mathrm{O}_{2}\right]^{+}$for the following process:

$$
\mathrm{O}_{2}^{-}+\mathrm{C}_{6} \mathrm{H}_{6} \rightarrow \mathrm{C}_{6} \mathrm{H}_{6} \mathrm{O}_{2}^{+}+2 e^{-}
$$

Differences in charge state and kinetic energy between reactions (1) and (2) are noticeable, but it is interesting to check if, from the theoretical point of view, reaction (2) is feasible in single collisions experiments. Molecular dynamics studies starting from the weakly bound $\left[\mathrm{C}_{6} \mathrm{H}_{6} \ldots \mathrm{O}_{2}\right]^{+}$complex, did not lead to formation of any stable compound with stoichiometry $\mathrm{C}_{6} \mathrm{H}_{6} \mathrm{O}_{2}{ }^{+}$(see details in the Supplementary Information). Thus, other mechanism should be responsible for the appearance of such cation in the TOF mass spectra. We first investigated the possibility of forming an electrostatically bonded complex by assuming an initial sudden double ionization of benzene by $\mathrm{O}_{2}^{-}$impact (see Fig. 4a), according to the reaction:

$$
\mathrm{O}_{2}^{-}+\mathrm{C}_{6} \mathrm{H}_{6} \rightarrow \mathrm{C}_{6} \mathrm{H}_{6}^{2+}+\mathrm{O}_{2}^{-}+2 e^{-} \rightarrow \mathrm{C}_{6} \mathrm{H}_{6} \mathrm{O}_{2}^{+}+2 e^{-}
$$

The resulting charged product, $\mathrm{C}_{6} \mathrm{H}_{6}{ }^{2+}$ and the projectile $\mathrm{O}_{2}{ }^{-}$will be attracted by an electrostatic force, thus leading a cationic complex of the form $\left[\mathrm{C}_{6} \mathrm{H}_{6} \ldots \mathrm{O}_{2}\right]^{+}$to be produced. Double ionization of benzene by electron impact has been recently studied by Wolff et al. ${ }^{25}$, showing an appearance energy of about $27 \mathrm{eV}$, which is in reasonably good agreement with the vertical double ionization energy of $24.6 \mathrm{eV}$, and the yield of formation, with respect to the parent ion, reaches a maximum value of about 5\% around $100 \mathrm{eV}$ electron impact energy. More recently Sigaud and Montenegro ${ }^{26}$ found this percentage to be higher than $10 \%$ and highlighted that benzene has a much larger double ionization cross section than other studied molecules ${ }^{26}$. In case of a $\mathrm{O}_{2}^{-}$projectile, this yield can be much more efficient. In fact, the energy spectrum of the beam transmitted through 1.3 mTorr of benzene, in Fig. $2 b$, shows an average energy loss with respect to the initial beam of about $16 \pm 3 \mathrm{eV}$. This demonstrates that the $700 \mathrm{eV}$ anion beam is able to excite, ionize or even double ionize benzene without losing the attached electron.

In order to describe the underlying molecular mechanism, several electronic excited states were computed in a frozen scan on the quasi-molecule $\left[\mathrm{C}_{6} \mathrm{H}_{6} \ldots \mathrm{O}_{2}\right]^{+}$. Figure $4 \mathrm{~b}$ shows the potential energy curves of the 250 computed electronic states as a function of the benzene-oxygen distance. States below $\sim 24 \mathrm{eV}$ (referred to the collision $\mathrm{O}_{2}{ }^{-}+\mathrm{C}_{6} \mathrm{H}_{6}$ ) lie under the second ionization threshold of benzene. Interestingly, we observe attractive 

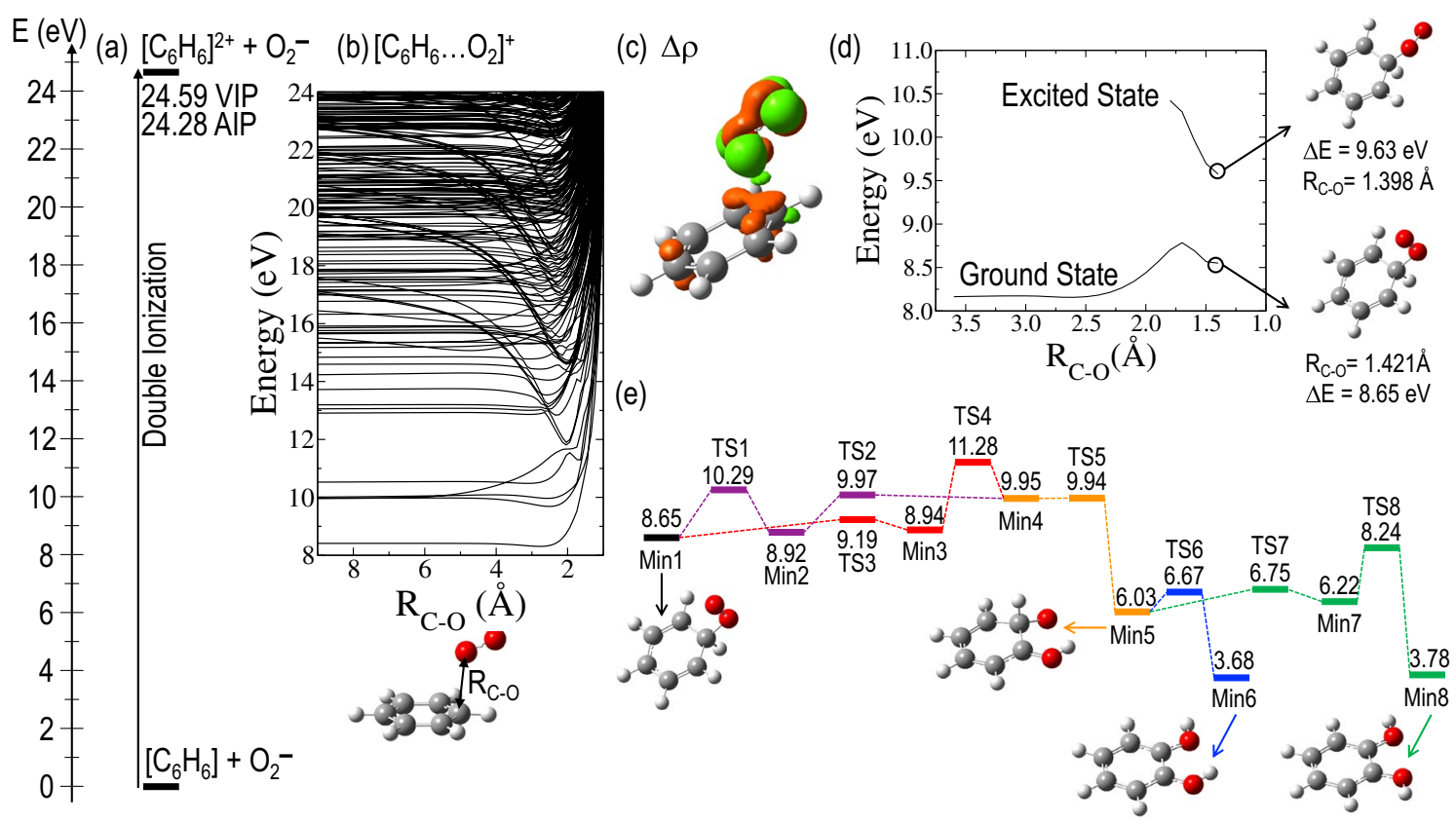

Figure 4. (a) $1 \mathrm{st}+2$ nd Ionization energy of benzene, $\mathrm{C}_{6} \mathrm{H}_{6}$, keeping the geometry of the neutral (vertical ionization potential-VIP), and after geometry optimization of the doubly-ionized (adiabatic ionization potential-AIP). (b) Potential energy curves as a function of the benzene-oxygen distance for the $\left[\mathrm{C}_{6} \mathrm{H}_{6} \ldots\right.$ $\left.\mathrm{O}_{2}\right]^{+}$system in a frozen scan. (c) Distribution of the electronic density difference ( $\Delta \rho$ with an isovalue of 0.007$)$ between the ground and the lowest energy excited state with charge transfer character $\mathrm{C}_{6} \mathrm{H}_{6}{ }^{2+}-\mathrm{O}_{2}$, for $\mathrm{R}_{\mathrm{C}-\mathrm{O}}$ $\approx 2 \AA$. Red and green lobes correspond to loss and gain of electron density, respectively. (d) Potential energy curves as a function of the $\mathrm{C}-\mathrm{O}$ distance in a relaxed scan both in the ground and in the excited states with charge transfer character. (e) Critical points in the potential energy surface of $\mathrm{C}_{6} \mathrm{H}_{6} \mathrm{O}_{2}^{+}$ground state with doublet spin multiplicity. Min indicates a minimum, TS indicates a transition state. Relative energies are given in $\mathrm{eV}$ and have been corrected with the zero-point-energy (ZPE). See rest of the structures in the Supplementary Information. In all panels relative energies are given in $\mathrm{eV}$ and refereed to the ground state of $\mathrm{C}_{6} \mathrm{H}_{6}$ and $\mathrm{O}_{2}{ }^{-}$at infinite distance.

curves, that correspond to a Coulomb-type attraction between the doubly positively charged benzene and the negatively charged oxygen in the entrance channel. These curves then collapse at a minimum close to $\sim 2 \AA$. In order to further our knowledge about the collision dynamics, a more refined examination of the potential energy surfaces has been implemented. To this, we have performed relaxed scans in the ground state, where the charge is redistributed over the whole $\left[\mathrm{C}_{6} \mathrm{H}_{6} \ldots \mathrm{O}_{2}\right]^{-}$system, and in the excited state of the lowest energy showing a charge transfer character, i.e. where it still presents the nature of the entrance channel with the superoxide anion approaching the doubly ionized benzene $\left[\mathrm{O}_{2}{ }^{-} \rightarrow \mathrm{C}_{6} \mathrm{H}_{6}{ }^{2+}\right]$. Figure $4 \mathrm{c}$ shows the difference in electron density distribution between these two states. A major change is appreciated with an increase of electron population of the $\mathrm{O}_{2}$ antibonding $\pi^{*}$ molecular orbital, which is filled by an electron from the $\mathrm{C}_{6} \mathrm{H}_{6}$ occupied $\pi$ molecular orbital. The Natural Transition Orbital (NTO) analysis shown in Fig. 5 confirms the charge transfer character of this state. The relaxed scans in both electronic states are shown in Fig. $4 \mathrm{~d}$, from which while in the excited state oxygen-carbon bonding appears as a barrier less process, a barrier of $\sim 0.7 \mathrm{eV}$ is shown in the ground state. Both calculations allow us to locate the corresponding minima in the potential energy surfaces, with strong covalent oxygen-carbon bonds stabilizing such structures, and the excited state showing charge transfer character. We can assume that non-radiative decay processes may be operative in such stabilization, since both states present similar structures and the corresponding potential energy curves are not far apart in energy, thus leading the system in the electronic ground state. It is precisely from this point where we continue our examination of the potential energy surface. Figure $4 \mathrm{e}$ shows the critical points, minima and transition states, in several reaction paths that lead to the production of stable diol structures (labeled as Min6 and Min8 in the figure). Energy barriers of $\sim 2-3 \mathrm{eV}$ in the forward direction, yielding diol structures, can be easily reached if we assume that the entrance channel is at higher energy. However, much higher energy barriers of $\sim 7-8 \mathrm{eV}$ prevent the diol structures to come back and to dissociate into $\mathrm{O}_{2} / \mathrm{C}_{6} \mathrm{H}_{6}$. Thus, these are kept trapped in these potential wells. Interestingly, these two diol structures appear only at $\sim 3.7 \mathrm{eV}$ above the initial point before the double ionization, i.e. $\mathrm{C}_{6} \mathrm{H}_{6}+\mathrm{O}_{2}{ }^{-}$. Such great stability seems to indicate that the $110 \mathrm{u}$ feature assigned to $\mathrm{C}_{6} \mathrm{H}_{6} \mathrm{O}_{2}{ }^{+}$most likely corresponds to any of these two diol compounds. Nevertheless, these structures may carry enough internal energy to continue evolving through low-energy barrier paths, e.g. with the loss of $\mathrm{OH}$; this is supported by the experiment where fragments $93 \mathrm{u}$ assigned to $\mathrm{C}_{6} \mathrm{H}_{5} \mathrm{O}^{+}$and/or $94 \mathrm{u}$ to $\mathrm{C}_{6} \mathrm{H}_{6} \mathrm{O}^{+}$are detected as combined features (see Fig. 3b) due 

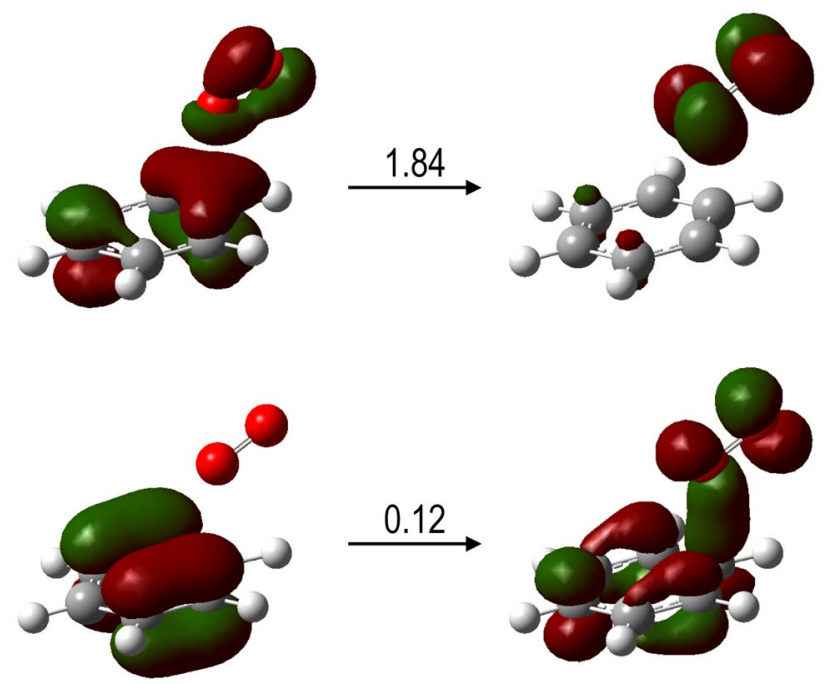

Figure 5. Natural transition orbitals (NTOs) analysis of the lowest energy excited state with charge transfer character $\mathrm{C}_{6} \mathrm{H}_{6}{ }^{2+}-\mathrm{O}_{2}$, for $\mathrm{R}_{\mathrm{C}-\mathrm{O}} \approx 2 \AA$. The two component transitions of this excited state as obtained with NTO are given with the corresponding coefficient, showing charge transfer from benzene to $\mathrm{O}_{2}$.

to the lack of mass resolution. Complementary molecular dynamics studies on the $\mathrm{C}_{6} \mathrm{H}_{6} \mathrm{O}_{2}{ }^{+}$compound (Min 1), confirm the stability of this structure, thus verifying the validity of the proposed mechanism (see details in the Supplementary Information). The formation of the complex should be a fast process taking place in a fs time scale. Considering strong coulomb attraction between doubly ionized benzene and $\mathrm{O}_{2}^{-}$, and assuming excitation energies of about $15-20 \mathrm{eV}$, a few tens of femtoseconds is enough to cleavage and form covalent bonds ${ }^{27,28}$.

It is worth mentioning in this point that benzene dication undergoes rearrangement to a more stable pyramidal isomer with a $\mathrm{C}_{5} \mathrm{H}_{5}$ base and $\mathrm{CH}$ at the apex ${ }^{29}$. It has been proposed that this structure is composed of a donor-acceptor bond from a $\mathrm{C}_{5} \mathrm{H}_{5}{ }^{-}$to a $\mathrm{CH}^{3+}$ moiety ${ }^{30}$. The isomerization barrier between the dicationic canonical form of benzene and the pyramidal one is of $100 \mathrm{~kJ} \cdot \mathrm{mol}^{-1}$, but we consider that in our case this possibility can be ruled out, since the Coulomb attraction between $\mathrm{C}_{6} \mathrm{H}_{6}{ }^{2+}$ and $\mathrm{O}_{2}{ }^{-}$leads to a very rapid formation of $\mathrm{C}_{6} \mathrm{H}_{6}-\mathrm{O}_{2}{ }^{+}$complex.

A careful analysis of the experimental mass spectra can provide additional information on the observed peak composition. Note that the time (mass) resolution of the present experiment $(\Delta m / m \cong 0.05)$ is limited by the gas cell configuration and the inherent characteristic of the hollow cathode discharge. Nonetheless, the decomposition of the observed peaks into single Gaussian functions, representing the different cationic fragment masses, may enlighten which ion species are contributing to the observed peaks. As mentioned above, Fig. $3 \mathrm{~b}$ shows the difference between the anion and electron induced fragmentation spectra once normalized to the parent ion $\left(\mathrm{C}_{6} \mathrm{H}_{6}{ }^{+}\right)$intensity, and therefore features around $78 \mathrm{u}$ do not appear in this plot. Similar conditions concur for the peaks around $50 \mathrm{u}$, i.e. $\mathrm{C}_{4} \mathrm{H}_{n}{ }^{+}(n=0-5)$ ion fragments. However, there is a clear enhancement of the peaks around $60 \mathrm{u}$ and $38 \mathrm{u}$, corresponding to $\mathrm{C}_{5} \mathrm{H}_{n}{ }^{+}(n=1-6)$ and $\mathrm{C}_{3} \mathrm{H}_{n}{ }^{+}(n=0-5)$, respectively, as well as new weaker structures appearing below $30 \mathrm{u}$. This suggests that anion collisions are favoring further parent ion fragmentation either by losing $\mathrm{CH}_{n}(n=0-4)$ fragments or breaking it into two similar size fragments.

For ions corresponding to $m / z>78 \mathrm{u}$, in which this study is mainly focused, we found the aforementioned $m / z=110-120 \mathrm{u}$ structure together with some smaller peaks around 94 and $99 \mathrm{u}$. The analysis of these structures using the decomposition into Gaussian functions reveals that $m / z=110-120 \mathrm{u}$ is formed by the contribution of two features centered at 110 and $126 \mathrm{u}$, respectively. The $110 \mathrm{u}$ is assigned to $\mathrm{C}_{6} \mathrm{H}_{6} \mathrm{O}_{2}{ }^{+}$with its formation mechanism supported by theoretical calculations described above. The $126 \mathrm{u}$ ion can be explained under the same formation rationale as the $110 \mathrm{u}$ feature but now being formed by the $\mathrm{O}_{3}{ }^{-}$anion, also present in the projectile beam, as: double ionization of benzene is followed by interactions between $\mathrm{O}_{3}{ }^{-}$and $\mathrm{C}_{6} \mathrm{H}_{6}{ }^{2+}$ leading to stable trihydroxybenzene (benzenetriol) structures $\mathrm{C}_{6} \mathrm{H}_{6} \mathrm{O}_{3}{ }^{+}$. $\mathrm{O}_{3}{ }^{-}$is also formed in the hollow cathode discharge and, although its time of flight from the cathode to the scattering cell is longer than $\mathrm{O}_{2}{ }^{-}$, the uncertainty in position where they are formed along the discharge contributes to a contamination of the primary $\mathrm{O}_{2}^{-}$beam (see FIG. 2a). Finally, we must note that the small feature at $94 \pm 2 \mathrm{u}$ confirms that the $\mathrm{C}_{6} \mathrm{H}_{6} \mathrm{O}_{2}^{+}$product may have enough internal energy to further decompose by losing $\mathrm{OH}^{*}$ or $\mathrm{O}^{*}$ radicals thus yielding $\mathrm{C}_{6} \mathrm{H}_{5} \mathrm{O}^{+}$or $\mathrm{C}_{6} \mathrm{H}_{6} \mathrm{O}^{+}$, respectively, as predicted by the quantum chemical calculations.

However, it is still surprising the high intensity of the $\mathrm{m} / z \sim 110-120 \mathrm{u}$ structure in comparison with $\mathrm{m} / z=78$ $\mathrm{u}$. Although double ionization of benzene has proven to be particularly likely ${ }^{17}$, single ionizing processes contributing to the $78 \mathrm{~m} / z$ peak should be even more pronounced. Nonetheless, this can be explained within our proposed model. Note that the Coulomb attraction between the formed cation and the anion projectile in the case of single ionization will give a neutral compound $\left(\mathrm{C}_{6} \mathrm{H}_{6} \mathrm{O}_{2}\right)$ which will not be detected by our TOF spectrometer but will contribute to reduce the observed $78 \mathrm{~m} / z$ peak intensity. Thus, an alternative way for the formation of the $110-120 \mathrm{~m} / z$ structure can be considered if the neutral $\mathrm{C}_{6} \mathrm{H}_{6} \mathrm{O}_{2}$ compound is formed in an excited autoionizing 


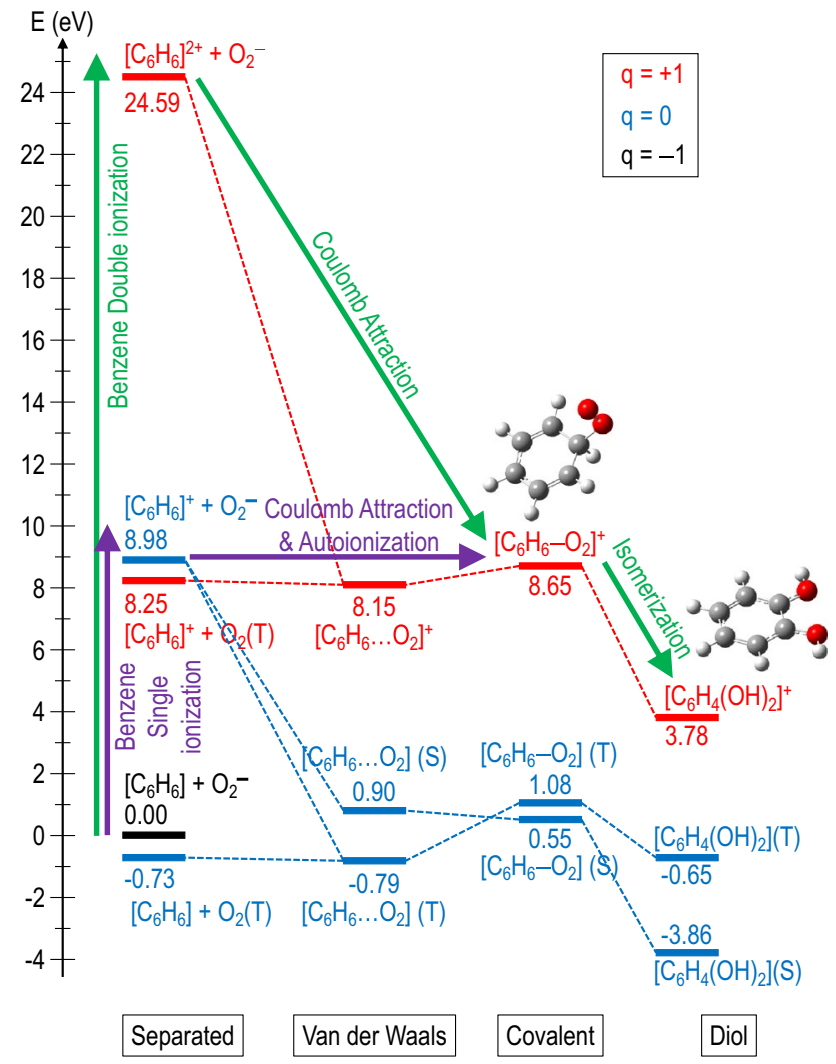

Figure 6. Different ways to produce neutral and positive charged benzene diols via $\mathrm{O}_{2}{ }^{-}$collisions with $\mathrm{C}_{6} \mathrm{H}_{6}$; -, single benzene ionization plus $\mathrm{O}_{2}{ }^{-}$Coulomb attraction to produce neutral diol configurations; - , same process but producing an autoionizing state which decay to the benzene diol cation. - , double benzene ionization plus Coulomb attraction leading to benzene diol cation formation.

state that finally decays to $\mathrm{C}_{6} \mathrm{H}_{6} \mathrm{O}_{2}{ }^{+}$, whose evolution would follow the potential energy surface shown in Fig. 4e. Note that the lifetime of this excited state will naturally contribute to form a broad structure as that shown here. In good agreement with the proposed model, the two pathways to form neutral and cation benzene diols via $\mathrm{O}_{2}^{-}$collisions with $\mathrm{C}_{6} \mathrm{H}_{6}$ are summarized in Fig. 6 .

\section{Conclusions}

In conclusion, we have experimentally shown the formation of large complex molecules (larger than the target molecule) in relatively high energy (200-900 eV) collisions of oxygen anions with neutral benzene molecules. Quantum chemical calculations have been performed within the framework of the density functional theory, to further our knowledge on the possible paths involved to generate these molecules. We have then proposed the formation of "quasi-molecular" compounds resulting from the electrostatic attraction between a doubly ionized target molecule and the negatively charged projectile. These electrostatically bonded complexes finally decay via non-radiative processes resulting in stable diol/triol structures in collisions with $\mathrm{O}_{2}{ }^{-} / \mathrm{O}_{3}{ }^{-}$. We have also shown that an alternative way to generate these complexes is to single ionize the benzene molecule, forming the "quasi-molecular" compound in an autoionizing state of its neutral configuration which finally decays to the corresponding diol cation. We can then confirm that the present experimental evidences are supported by the theoretical calculations. This model presents a new high energy anion-driven chemistry as an alternative way to form complex molecules.

Received: 29 September 2021; Accepted: 16 November 2021

Published online: 30 November 2021

\section{References}

1. Costa, F. et al. A complete cross section data set for electron scattering by pyridine: Modelling electron transport in the energy range 0-100 eV. Int. J. Mol. Sci. 21, 6947 (2020).

2. Bak, A. et al. Novel benzene-based carbamates for AChE/BChE inhibition: Synthesis and ligand/structure-oriented SAR study. Int. J. Mol. Sci. 20, 1524 (2019).

3. Matsui, K., Segawa, Y. \& Itami, K. All-benzene carbon nanocages: size-selective synthesis, photophysical properties, and crystal structure. J. Am. Chem. Soc. 136, 16452-16458 (2014). 
4. Silveira, J. F. R. V. \& Muniz, A. R. Functionalized diamond nanothreads from benzene derivatives. Phys. Chem. Chem. Phys. 19, 7132-7137 (2017).

5. Marx, J. Oxygen free radicals linked to many diseases. Science 235, 529-531 (1987).

6. Hayyan, M., Hashim, M. A. \& AlNashef, I. M. Superoxide Ion: Generation and Chemical Implications. Chem. Rev. 116, 3029-3085 (2016).

7. Shigdel, U. K. et al. The trajectory of intrahelical lesion recognition and extrusion by the human 8-oxoguanine DNA glycosylase. Nat. Commun. 11, 4437 (2020).

8. Norrish, R. G. W. \& Taylor, G. W. The oxidation of benzene. Proc. R. Soc. London. Ser. A. Math. Phys. Sci. 234, 160-177 (1956).

9. Lay, T. H., Bozzelli, J. W. \& Seinfeld, J. H. Atmospheric Photochemical Oxidation of Benzene: Benzene + $\mathrm{OH}$ and the Benzene-OH Adduct (Hydroxyl-2,4-cyclohexadienyl) + O 2. J. Phys. Chem. 100, 6543-6554 (1996).

10. Battistel, E., Tassinari, R., Fornaroli, M. \& Bonoldi, L. Oxidation of benzene by molecular oxygen catalysed by vanadium. J. Mol. Catal. A Chem. 202, 107-115 (2003).

11. Shiota, Y., Suzuki, K. \& Yoshizawa, K. Mechanism for the Direct Oxidation of Benzene to Phenol by FeO +. Organometallics 24, 3532-3538 (2005).

12. Ascenzi, D., Franceschi, P., Guella, G. \& Tosi, P. Phenol Production in Benzene/Air Plasmas at Atmospheric Pressure. Role of Radical and Ionic Routes. J. Phys. Chem. A 110, 7841-7847 (2006).

13. Larsson, M., Geppert, W. D. \& Nyman, G. Ion chemistry in space. Reports Prog. Phys. 75, 066901 (2012).

14. Mendes, M. et al. Experimental electron-detachment cross sections for collisions of O2- with N2 molecules in the energy range $50-7000$ eV. Phys. Rev. A 99, 062709 (2019).

15. NIST Chemistry WebBook. https://webbook.nist.gov/chemistry. Available online: https://webbook.nist.gov/chemistry

16. Yanai, T., Tew, D. P. \& Handy, N. C. A new hybrid exchange-correlation functional using the Coulomb-attenuating method (CAMB3LYP). Chem. Phys. Lett. 393, 51-57 (2004).

17. Dunning, T. H. Gaussian basis sets for use in correlated molecular calculations. I. The atoms boron through neon and hydrogen. J. Chem. Phys. 90, 1007-1023 (1989).

18. Guido, C. A., Knecht, S., Kongsted, J. \& Mennucci, B. Benchmarking Time-Dependent Density Functional Theory for Excited State Geometries of Organic Molecules in Gas-Phase and in Solution. J. Chem. Theory Comput. 9, 2209-2220 (2013).

19. Mahato, B. \& Panda, A. N. Assessing the Performance of DFT Functionals for Excited-State Properties of Pyridine-Thiophene Oligomers. J. Phys. Chem. A 125, 115-125 (2021).

20. Bauernschmitt, R. \& Ahlrichs, R. Treatment of electronic excitations within the adiabatic approximation of time dependent density functional theory. Chem. Phys. Lett. 256, 454-464 (1996).

21. Casida, M. E., Jamorski, C., Casida, K. C. \& Salahub, D. R. Molecular excitation energies to high-lying bound states from timedependent density-functional response theory: Characterization and correction of the time-dependent local density approximation ionization threshold. J. Chem. Phys. 108, 4439-4449 (1998).

22. Martin, R. L. Natural transition orbitals. J. Chem. Phys. 118, 4775 (2003).

23. Frisch, M. J. et al. Gaussian 16, Revision C.01. (2016).

24. Tubaro, M., Marotta, E., Seraglia, R. \& Traldi, P. Atmospheric pressure photoionization mechanisms. 2. The case of benzene and toluene. Rapid Commun. Mass Spectrom. 17, 2423-2429 (2003).

25. Wolff, W. et al. Production of Long-Lived Benzene Dications from Electron Impact in the 20-2000 eV Energy Range Combined with the Search for Global Minimum Structures. J. Phys. Chem. A 124, 9261-9271 (2020).

26. Sigaud, L. \& Montenegro, E. C. Absolute cross sections for production of molecular dications by electron impact. J. Phys. Conf. Ser. 1412, (2020).

27. Maclot, S. et al. Dynamics of glycine dications in the gas phase: Ultrafast intramolecular hydrogen migration versus Coulomb repulsion. J. Phys. Chem. Lett. 4, 3903-3909 (2013).

28. Maclot, S. et al. Determination of Energy-Transfer Distributions in Ionizing Ion-Molecule Collisions. Phys. Rev. Lett. 117, 1-6 (2016).

29. Jašík, J., Gerlich, D. \& Toithová, J. Probing Isomers of the Benzene Dication in a Low-Temperature Trap. J. Am. Chem. Soc. 136, 2960-2962 (2014).

30. Fantuzzi F., Oliveira de Sousa D. W., Chaer Nascimento M. A. Chemical bonding in the pentagonal-pyramidal benzene dication andanalogous isoelectronic hexa-coordinate species. Comput. Theor. Chem.1116, 225-233 (2017).

\section{Acknowledgements}

This study is partially supported by the Spanish Ministerio de Ciencia e Innovación (Projects PID2019-104727RBC21 and PID2019-110091GB-I00 funded by MCIN/AEI/10.13039/501100011033), the 'María de Maeztu' (CEX2018-000805-M) Program for Centers of Excellence in R\&D, and CSIC (Project LINKA20085). This article is based upon work from COST action CA18212-Molecular Dynamics in the GAS phase (MD-GAS), supported by COST (European Cooperation in Science and Technology). The authors acknowledge the generous allocation of computer time at the Centro de Computación Científica at the Universidad Autónoma de Madrid (CCC-UAM). CG acknowledges funding from the the IFHARU-SENACYT Program of Panamá through the Grant No. 270-2018-364. SK acknowledges the Portuguese National Funding Agency FCT through the Grant PD/BD/142831/2018 and together with PLV the Research Grants CEFITEC (UIDB/00068/2020) and PTDC/ FIS-AQM/31281/2017. This work was also supported by the Radiation Biology and Biophysics Doctoral Training Programme (RaBBiT; Grant PD/00193/2012) and UCIBIO (Grant UIDB/04378/2020).

\section{Author contributions}

PLV and GG conceived and conducted the experiment, CG, MM and AIL did the measurements, SDT and FAG did the calculations, FAG, SDT, PLV and GG analyzed the data. All the authors reviewed the manuscript.

\section{Funding}

IFHARU-SENACYT Panamá,270-2018-364, Portuguese National Funding Agency FCT,PD/ BD/142831/2018,PTDC/FIS-AQM/31281/2017,PTDC/FIS-AQM/31281/2017, Maria de Maeztu Program for Centers of Excellence in R\&D,CEX2018-000805-M,Ministerio de Ciencia e Innovación MCIN/ AEI/10.13039/501100011033, PID2019-110091 GB-I00,PID2019-104727RB-C21,CEFITEC Portugal,UIDB/0 0068/2020,UIDB/00068/2020,UIDB/00068/2020.

\section{Competing interests}

The authors declare no competing interests. 


\section{Additional information}

Supplementary Information The online version contains supplementary material available at https://doi.org/ 10.1038/s41598-021-02408-7.

Correspondence and requests for materials should be addressed to S.D.-T. or G.G.

Reprints and permissions information is available at www.nature.com/reprints.

Publisher's note Springer Nature remains neutral with regard to jurisdictional claims in published maps and institutional affiliations.

(c) (i) Open Access This article is licensed under a Creative Commons Attribution 4.0 International License, which permits use, sharing, adaptation, distribution and reproduction in any medium or format, as long as you give appropriate credit to the original author(s) and the source, provide a link to the Creative Commons licence, and indicate if changes were made. The images or other third party material in this article are included in the article's Creative Commons licence, unless indicated otherwise in a credit line to the material. If material is not included in the article's Creative Commons licence and your intended use is not permitted by statutory regulation or exceeds the permitted use, you will need to obtain permission directly from the copyright holder. To view a copy of this licence, visit http://creativecommons.org/licenses/by/4.0/.

(C) The Author(s) 2021 